\title{
The third release of the Large Quasar Astrometric Catalog (LQAC-3): a compilation of 321957 objects ${ }^{\star}$
}

\author{
J. Souchay ${ }^{1}$, A. H. Andrei ${ }^{1,2,3}$, C. Barache ${ }^{1}$, T. Kalewicz ${ }^{1}$, C. Gattano ${ }^{1}$, B. Coelho ${ }^{3}$, \\ F. Taris ${ }^{1}$, S. Bouquillon ${ }^{1}$, and O. Becker ${ }^{1}$ \\ ${ }^{1}$ Observatoire de Paris, SYRTE/UMR-8630 CNRS/UPMC, 75014 Paris, France \\ e-mail: Jean. Souchay@obspm.fr \\ 2 Observatorio Nacional/MCT, 20921-400 Rio de Janeiro, Brazil \\ 3 Observatorio do Valongo, UFRJ, 20080-090 Rio de Janeiro, Brazil
}

Received 13 March 2015 / Accepted 18 June 2015

\section{ABSTRACT}

\begin{abstract}
Context. From an astrometric point of view, quasars constitute quasi-ideal reference objects in the celestial sphere, with an a priori absence of proper motion. Since the second release of the Large Quasar Astrometric Catalog (LQAC), a large number of quasars have been discovered, in particular with the upcoming new release of the SDSS quasars catalog.

Aims. Following the same procedure as in the two previous releases of the LQAC, our aim was to compile all the quasars recorded until the present date, with accurate recomputation of their equatorial coordinates in the ICRS and with the maximum of information concerning their physical properties, such as the redshift, the photometry, and the absolute magnitudes.

Methods. For the purpose above, we carried out the cross-identification between the 9 catalogs of quasars chosen for their huge number of objects, including all the available data related to magnitudes, radiofluxes, and redshifts. This cross identification was particularly delicate because of a slight change in coordinates between the objects common to two successive releases of the SDSS and the elimination of some of them. Equatorial coordinates were recomputed more accurately according to the algorithms used for the elaboration of the Large Quasar Reference Frame (LQRF). Moreover, absolute magnitudes and morphological indexes of the new objects were given, following the same method as in the LQAC-2.

Results. Our final catalog, called LQAC-3, contains 321957 objects including a small proportion of AGNs (14 128) and BLLac (1183). This is roughly $70 \%$ more than the number of objects recorded in the LQAC-2.

Conclusions. The LQAC-3 will be useful for the astronomical community since it gives the most complete information available about the whole set of already recorded quasars, with emphasis on the precision and accuracy of their coordinates with respect to the ICRF2.
\end{abstract}

Key words. catalogs - astrometry - reference systems - quasars: general - astronomical databases: miscellaneous

\section{Introduction}

Quasars have become fundamental objects both from an astrophysical and an astrometric point of view. First they are necessary for our understanding of galaxy evolution and of the expansion of the universe. At the same time, they constitute quasi-ideal geometric reference sources in the celestial sphere, because of the absence of proper motion even at the submilliarcsecond level of position obtained by modern techniques such as Very Long Baseline Interferometry (VLBI). For these reasons, there has been continuous effort for almost three decades by Véron-Cetty \& Véron $(1984,2010)$ to construct an overall compilation of all the quasars detected so far. In their last release in 2010, the 13th one, their catalog included 133336 quasars and 34231 active galaxies.

With the same aim, Souchay et al. (2009, 2012) constructed two successive versions of a compilation of quasars called Large Quasar Astrometric Catalog (LQAC). The last one, called LQAC-2, contains 188504 objects. Véron-Cetty \& Véron (2010) have contested that the LQAC is not, strictly speaking, a quasar catalog because several of the included objects (2921) have no optical identification or measured redshift. This is itself

* The catalog is available at the CDS via anonymous ftp to cdsarc.u-strasbg. fr $(130.79 .128 .5)$ or via http://cdsarc.u-strasbg.fr/viz-bin/qcat?]/A+A/583/A75 contestable. Indeed, most of these last objects are categorized as quasars by the VLBI community for their very pointlike aspect and very accurate coordinates at radio wavelengths bands (generally $\mathrm{S}$ and $\mathrm{X}$ ). In consequence they satisfy the original definition of "quasi stellar source", even if their redshift is not systematically available.

Moreover, the definition of a quasar has not been settled very well. For instance, we notice that in their studies, Véron-Cetty \& Véron (2010) define a quasar in a purely arbitrary manner as a starlike object or an object with a starlike nucleus that is brighter than $M_{B}=-22.25$, assuming a well established cosmology. Moreover they recognize that the limit between quasars and active galactic nuclei (AGNs) is far from being obvious in some cases. According to their definition, some objects would move from QSO to AGN and vice versa if the apparent magnitude $B$ becomes more precise or if cosmological parameters for calculating $M_{B}$ as $H_{0}, q_{0}$ change slightly.

In a different way, in the two recent versions DR9Q (Pâris et al. 2012) and DR10Q (Pâris et al. 2014) of the SDSS quasar catalog, a quasar is also arbitrarily defined as an object with luminosity $M_{i}[z=2] \leq-20.5$, which either displays at least one emission line with $F W M H \geq 500 \mathrm{~km} \mathrm{~s}^{-1}$ or, if not, presents significant absorption features that characterize a quasar without ambiguity thanks to the Lyman- $\alpha$ forest or to BAL troughs.

To avoid the rigid (and not physically satisfactory) definitions of quasars above, as well as to avoid the uncertainty in 
the status of the objects, we decided in this paper, as in the LQAC-2, to include in the LQAC-3 the objects that satisfy the conditions either of Véron-Cetty \& Véron or those of the SDSS mentioned above, and also compact extragalactic objects as the AGN's recorded in Véron-Cetty \& Véron (2010). Nevertheless we give for each object of our compilation information that allows the user to have the possibility of categorizing it as a quasar according to our considerations. This is why, in addition to usual data, such as magnit udes and redshift, we give complementary information, such as the absolute magnitudes at two wavelengths $M_{B}$ and $M_{I}$ and morphology indexes (sharpness, skewness, roundness).

\section{Inclusion of the recent release of the SDSS quasar catalog}

All the new quasars included in LQAC-3 and not in LQAC-2 come exclusively from the SDSS, and for a large proportion of the last version DR10Q of the SDSS-III. The DR10Q itself has been constructed starting from the Baryon Oscillation Spectroscopic Survey (BOSS) of the SDSS-III. BOSS is based on imaging released in SDSS-DR8 (Aihara et al. 2011), which corresponds to the SDSS-I/II with an extension to the South Galactic CAP (SGC). The DR10Q was built from visual inspection of the spectra of all the objects targeted as quasars by SDSSIII/BOSS, together with objects clearly satisfying $z \geq 2$ by the SDSS pipeline among galaxy targets (Bolton et al. 2012). The visual inspection process is described in detail in Pâris et al. (2012). Starting from an initial list of 316947 quasar targets that were visually inspected, 166583 objects have been identified as QSOs. Then, 545 supplementary ones were clearly classified as QSOs but with an uncertain value of the redshift, whereas 1370 ones present good arguments to definitely be considered as quasars. These two last categories have not been taken into consideration in our sample.

In fact our total SDSS sample of quasars belonging to our LQAC-3 catalog comes from three different sources: the DR7Q catalog (Schneider et al. 2010) containing 105783 objects, the DR10Q (Pâris et al. 2014) containing 166583 objects, and the remaining SDSS objects not included in these two last versions but already recognized as SDSS quasars already in the last version of the Véron-Cetty \& Véron (2010) catalog. Considering that 16420 objects were shared with the DR7Q and DR10Q catalogs must be counted only once, our total number of SDSS quasars finally reaches 262535 objects, which is more than twice the amount of SDSS quasars in the LQAC-2 catalog (Souchay et al. 2012).

\section{Cross-identifications between catalogs}

Because the SDSS is by far the leading catalog included in the LQAC, as well as the exclusive contributor of the new objects belonging to the third version of the LQAC with respect to the second one, we find it judicious to give the information concerning both the cross-identification between the LQAC-2 and LQAC-3 in the following, as well as an updated study of common quasars between the SDSS and the other catalogs present in the LQAC compilation.

\subsection{Cross-identifications with the LQAC-2}

As already explained in a preceding section, the quasars present in the LQAC-3 and missing in the LQAC-2 correspond exclusively to objects that were newly identified in the recent updates of the SDSS, namely the DR7Q-DR10Q compilation. Thus the addition of these new quasars may be considered as a single and direct task, with no particular difficulty. In fact, this task is more complicated than expected for two reasons. First, the coordinates of all the SDSS objects have changed from the DR8 release to which the LQAC-2 is referred, to the DR7Q-DR10Q combined release to which the LQAC-3 is related. Second, some of the SDSS quasars present in the LQAC-2 were no longer included in our SDSS selected sample for the LQAC-3, because their status as quasars was finally rejected (Pâris, priv. comm.) as explained below.

Thus starting from the 187504 objects of the LQAC-2, only 43870 have all their data (coordinates, redshift, photometry, etc.) that remained unchanged because they do not belong to the SDSS. Among the 143634 remaining SDSS objects, 1650 have been eliminated in the LQAC-3 for they are no longer included in the combined DR7Q-DR10Q release of the SDSS, although they were present in the SDSS release of the LQAC-2. They must be considered as outliers, mainly stars, ejected after a meticulous spectroscopic check. Finally this left us with a set of 141984 SDSS quasars whose the equatorial coordinates have systematically changed. For these quasars a cross-identification was essential, in particular for attributing the same LQAC number in both the LQAC-2 and LQAC-3. Generally, the coordinates' differences both in right ascension and declination, at the level of 0 .' 1 or less do not permit any ambiguity in the correspondence between the quasars shared by the LQAC-2 and LQAC-3.

\subsection{Cross-identification between SDSS and other catalogs}

The predominant weight of the quasars of the SDSS catalog in the total LQAC-3 sample leads to the natural question of their membership in the other constituent catalogs of the compilation. In Table 1 we present the number of the common quasars. We find only 506 sources in common with the ICRF2 catalog, which represents a mere $14.8 \%$ of the ICRF2. This is explained easily for two: first the ICRF2 has its 3414 sources regularly dispatched in the whole celestial sphere, ensuring an all-sky coverage, whereas the SDSS survey, regardless of the release concerned is concentrated in a limited area of the sky, roughly one-third. Second, the sources included in the ICRF2 catalog are observed in VLBI radio bands, namely $\mathrm{S}$ and $\mathrm{X}$, which does not guarantee that they have an optical counterpart bright enough to be detected with the SDSS pipeline. This also remark prevails for the three other radio loud quasars of the VLBA (B), the VLA (C), and the JVAS (D), with relative common detection ratios of $13.2 \%, 19.2 \%$, and $21.3 \%$.

\section{Celestial reference frame coordinates}

In addition to delivering the equatorial coordinates coming from the original compiled catalogs, the LQAC-3 is constructed to offer the most precise available astrometric equatorial coordinates for each of the quasars included. This fulfills the goal of unmistakably finding any object without introducing position ambiguities.

At the same time, the large number of quasars present in the LQAC-3 catalog enables the opposite to be equally served: once their position has been optimized, the overall sample of quasars included can be considered as a realization of the International Celestial Reference System (ICRS) in the optical band, i.e. a secondary representation of the International Celestial Reference Frame (ICRF), defined by Very-long base interferometry in the 
Table 1. Number of quasars for each catalog of the LQAC-3 compilation with the corresponding number of quasars in common with the SDSS catalog.

\begin{tabular}{lllrr}
\hline \hline $\begin{array}{l}\text { Catalog } \\
\text { name }\end{array}$ & Flag & Nature & $\begin{array}{r}\text { Nbs of } \\
\text { quasars } \\
\text { (LQAC-3) }\end{array}$ & $\begin{array}{r}\text { Nb. of } \\
\text { quasars } \\
\text { common } \\
\text { with SDSS }\end{array}$ \\
\hline ICRF2 & A & radio & 3414 & 506 \\
VLBA & B & radio & 7213 & 951 \\
VLA & C & radio & 1858 & 356 \\
JVAS & D & radio & 2118 & 451 \\
2QZ & F & optical & 23660 & 3271 \\
2df-SDSS LRG & G & optical & 9058 & 1923 \\
FIRST & H & radio & 969 & 801 \\
HB & I & opt. and radio & 6720 & 2102 \\
2MASS & J & infrared & 21884 & 18433 \\
GSC2.3 & K & optical & 144508 & 113213 \\
B1.0 & L & optical & 139192 & 111121 \\
V\&V & M & optic. and radio & 79692 & 25191 \\
\hline
\end{tabular}

radio domain. For the purpose of the astrometric optimization above, we follow the same steps as in the construction of the Large Quasar Reference Frame, the LQRF and LQRF2 (Andrei et al. 2009; Souchay et al. 2012). They are summarized below.

Since the original optical coordinates come from several surveys, generally non-astrometric ones, a preliminary adjustment to the J2000 standard is made when necessary. Successive steps are then undertaken to derive equatorial ICRF aligned coordinates. First, the optical counterparts of the quasars are retrieved in large deep catalogs: the USNO B1.0 (Monet et al. 2003), the GSC2.3 (Lasker et al. 2008), and SDSS.Then a corresponding local neighborhood centered on the quasar is aligned to the UCAC reference frame (Zacharias et al. 2004), which is itself linked to the HIPPARCos celestial frame. Next, a large sample of quasars with radio positions directly tied to the ICRF is selected. The sample is formed by the ICRF sources themselves, plus the VLBA and VLA calibrators. The precision of the radio astrometric coordinates is at the milli-arcsecond level or better. Thus the systematic difference with the optical coordinates can be used to derive global corrections to the latter. This assumes that the optical-to-radio displacement of centroids has no preferred direction and is negligible on average and on a global scale. Such a sample of quasars with optical coordinates tied to the UCAC frame and radio coordinates tied to the ICRF is henceforth used to derive global global corrections to the optical coordinates of all the quasars. The slow rotation of the HIPPARCos frame and misalignments of the UCAC frame are corrected by deriving the direction cosines of the rotation matrix plus an equatorial translation. Recurrent zonal patterns are represented and corrected using spherical harmonic functions. Zonal warps are corrected by using average differences obtained in large, numerically representative spherical caps. At all steps, magnitude and color corrections were also considered.

This process was followed for the sources already present in the LQAC-2 edition. Since the optical positions are the same and the ICRF orientation has not changed, the corresponding prior LQRF positions have also been maintained. As explained in an earlier section, the new optical sources in the present LQAC-3 edition come exclusively from new SDSS Data release. For these the same process as described above was followed, except the first step, namely the local adjustment to the UCAC frame. The reason is that the former versions of the LQRF (Andrei et al. 2009) verified that the precision and accuracy of the UCAC and

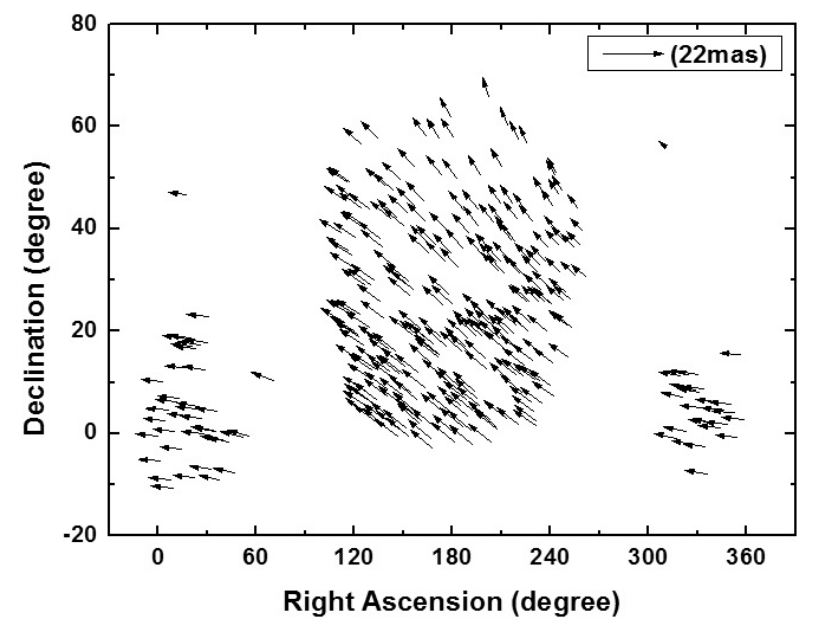

Fig. 1. Vector map corresponding to the equatorial coordinate variation caused by the global rotations away from the SDSS quasar frame to the ICRF2. Owing to the very large number of sources, only 314 are represented, drawing the 1:1000 sample randomly (the systematic nature of the rotations is clearly seen).

SDSS catalogs are comparable. The SDSS positions were kept as a starting point for the following steps of global corrections, thus avoiding the introduction of spurious terms of local adjustment, magnitude, and color.

The direction cosines around the ICRF axis Ox and Oy (old equinox and contra-equinox directions) are small. On the other hand, the corrections to the origins of right ascension and declination are significant: their values are $-9.1 \pm 1.8$ mas, and $4.1 \pm 2.0$ mas. respectively.

The three rotations (optical minus radio, positive, anticlockwise) and the equatorial translation were applied and the spherical harmonic procedures then followed. Results can be seen in Fig. 1. Recurrent dependences on right ascension, declination, and magnitude were probed for. As a result the zonal terms are found to not be significant, and no corresponding correction was applied. None of the terms is at the same time larger than 50 mas and significant above $3 \sigma$. The terms are larger in declination, probably because of the uneven latitudinal distribution. In right ascension the high frequency terms are more important, probably because they are better determined in the high-density, but space-confined, DR10Q distribution.

The magnitude terms are also small and do not present any significant behavior, indicating that the magnitude equation present in earlier DR version is very mitigated for the DR10Q. Finally, systematic local deviations from the original equatorial coordinates are calculated as the average between the radio and optical counterparts of the sources common to the ICRF and the DR10Q. We require at least five sources to calculate the average. This is easily attained in a small radius within the densest part of the SDSS, but the radius must be taken as progressively larger in the most recent, less populated regions of the DR10Q (Fig. 2).

As expected, the systematic corrections, as given by the total averages, are small ( -0.9 mas in right ascension. and 1.0 mas in declination), nevertheless the resulting average displacement is $54.5 \pm 12.8$ mas (Fig. 3).) The average radius of the circles within which the averages were calculated was $4.7^{\circ}$, and the distribution of the standard deviations to the mean values is markedly Poisson-like with a peak at 14.7 mas. The combination of corrections for the global rotation and for the systematic local deviations is effective, and the mean distance between the ICRF and LQRF positions is slashed to $21.6+/-13.4$ mas. 


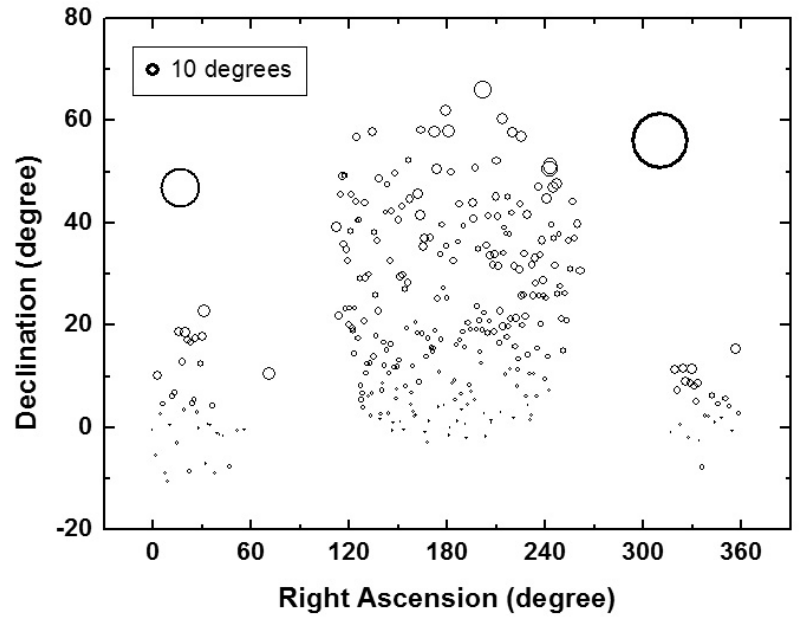

Fig. 2. Graphic representation of the neighborhoods in which the remaining local zonal position corrections are removed. The mean neighborhood is $4.7 \mathrm{deg}$, and there are only $13.6 \%$ neighborhood radii larger than $6 \mathrm{deg}$. As before, owing to the large number of quasars, the sample is a representation 1:1000, to 314 elements (since the SDSS is tightly packed, this representation remains adequate).

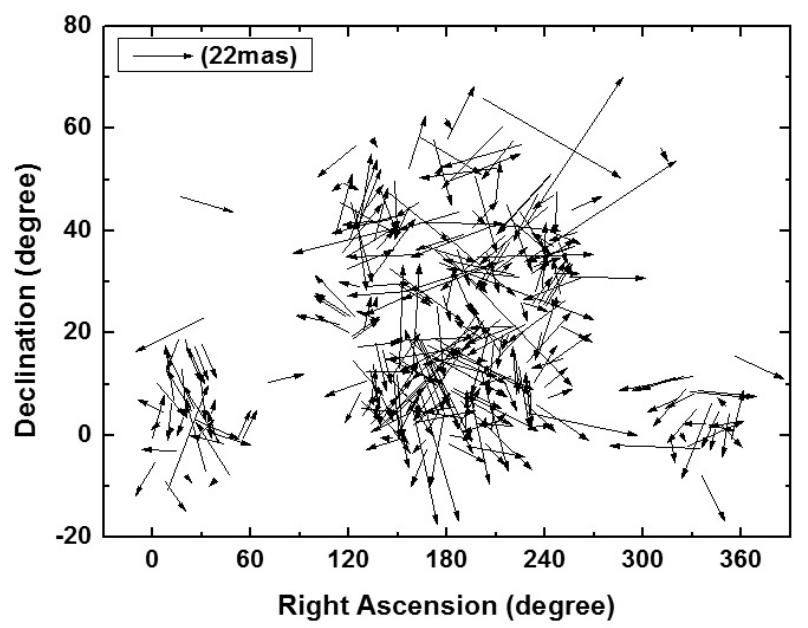

Fig. 3. Vector map corresponding to the local zonal position corrections. As before, the whole population of quasars is sampled at rate 1:1000. The main text discusses the removal of global rotations and the absence of significant recurrent zonal terms.

The first two releases of the Gaia catalog, scheduled for 2016 and 2017, will contain objects with acceptable formal standard errors of positions. Thanks to the faintness of its objects, the LQRF will therefore keep on providing a direct realization of the ICRS in the optical window after this term. Examples of it usefulness are related to the Gaia mission, to better locate targets of opportunity either spotted by Gaia or instead to be searched among the vast number of Gaia single observations. Naturally in later issues, the catalog of quasars provided by Gaia will supersede the LQRF. The latter will still be useful for providing a confirmation of the alignment to the ICRF, which will be done directly by Gaia (Bourda et al. 2011) and independently in the LQRF via star catalogs.

\section{Determination of absolute magnitudes}

Given the piecewise information available for quasars, because of the enormous distances, absolute magnitudes are key parameters for modeling masses and sizes. In the present version, we follow the same approach as in the previous versions of the LQAC by determining the absolute magnitudes. On that, the apparent magnitude is scaled up by the distance, minus the extinction, whereas a correction factor compensates for the relativistic doppler shift. The expression below results in

$M=m+5-5 \log D_{\mathrm{L}}-A-K$

where $M$ and $m$ are respectively the absolute and apparent magnitudes of the quasars at a given bandpass, $D_{\mathrm{L}}$ is the luminosity distance, $A$ represents the galactic extinction, and $K$ (usually termed as $K$-correction) is the effect of the redshift on the part of the spectrum corresponding to the filter through which $M$ is determined.

The infrared attenuation atmospheric gap makes this window favorable for observing faint sources. For quasar detectability, the infrared has the added advantage of being free of Lyman-alpha absorption up to high redshifts, and on the other hand, it is a relatively line-free region at the rest frame of emission. As a result, the I magnitude is often used in massluminosity and mass-radius model relationships (e.g., Shen et al. 2008; Morgan et al. 2010). On the opposite side of the vicinity of the optical spectral window, $B$ absolute magnitudes sit at the top of the blue-bump emission enhancement, which is crucial for the well known blue excess that distinguishes quasars from the stellar population. The $I$ and $B$ apparent magnitudes are readily available for the LQAC-3 sources from the DSS and SDSS photometric measurements, and the corresponding absolute magnitudes are presented.

According to Hogg et al. (2000), in the case of an homogeneous and isotropic cosmological model with a FriemannLemaître-Robertson-Walker metrics and a null space curvature, the expression of the luminosity distance $D_{\mathrm{L}}$ of a quasar with redshift $z$ is given by

$D_{\mathrm{L}}=\frac{(1+z) c}{H_{0}} \int_{1}^{1+z} \frac{\mathrm{d} u}{\sqrt{\frac{1}{3}\left(1-2 q_{0}\right)+\frac{2}{3}\left(1+q_{0}\right) u^{3}}}$

where $c$ is the speed of light, $H_{0}$ the Hubble expansion factor, and $q_{0}$ a deceleration parameter. The dependence of the luminosity distance $D_{\mathrm{L}}$ on the values of $q_{0}$ and $H_{0}$ was discussed by Souchay et al. (2009). In this paper, the values of the fundamental parameters were $q_{0}=-0.58$ and $H_{0}=$ $72 \mathrm{~km} \mathrm{~s}^{-1} \mathrm{Mpc}^{-1}$, which are consistent with recent cosmological constraints (Komatsu et al. 2011). Here we calculated the luminosity distance using slightly different values for these cosmological constraints. We used $H_{0}=70 \mathrm{~km} \mathrm{~s}^{-1} \mathrm{Mpc}^{-1}, \Omega_{\mathrm{M}}=0.3$, $\Omega_{\lambda}=0.7$, and $q_{0}=-0.65$.

The galactic reddening is extracted from the $E(B-V)$ extinction maps of Schlegel et al. (1998). The interpolation uses the code from the same authors. The $K$ corrections are those from Richards et al. (2006) for the infrared band, and from Véron-Cetty \& Véron (2006) for the blue band. Input values of the apparent magnitudes are from the GSC2.3, the PPMXL, and the DR10Q SDSS catalogs.

Figure 4 shows the distribution of the mean apparent magnitudes per bin of redshift. As a general feature, the higher the redshift, the dimmer the brightness because of the increasing distance. Moreover, for the same source at the same distance, the $B$ magnitudes are larger than the $I$ magnitudes because of atmospheric extinction.

On the other hand, Fig. 5 shows the distribution of the mean absolute magnitudes per bin of redshift $z$. Up to $z=5$, the increasing QSO activity is seen, mitigated in the figure by the cosmological correction from luminosity distance to redshift. For 


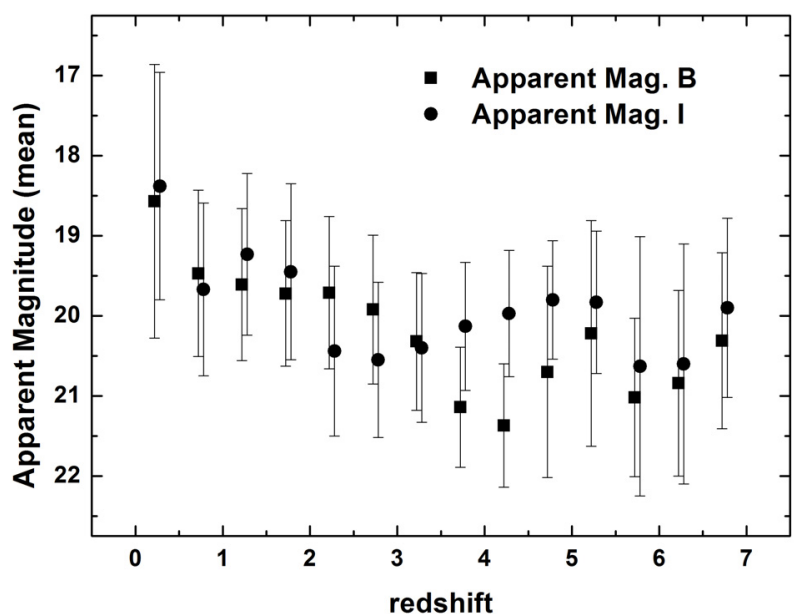

Fig. 4. Distribution of the mean apparent magnitudes per bin of redshift. Symbols are slightly offset but refer to the same redshift bin.

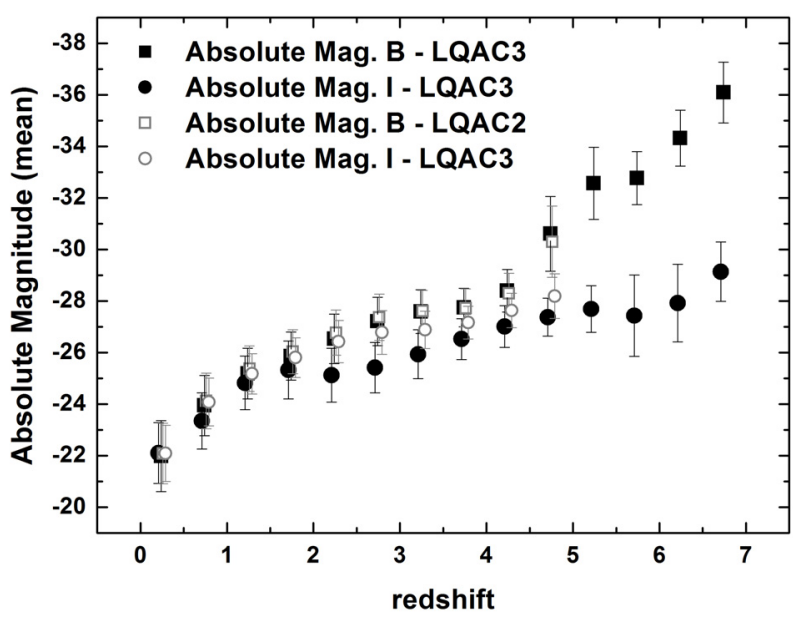

Fig. 5. Distribution of the mean absolute magnitudes per bin of redshift. Only sources for which both $B$ and $I$ absolute magnitudes were determined are considered to the mean. Also shown is the same distribution as for the sources in the earlier version LQAC2 - that is, without the SDSS BOSS sources - and no net variation appears. The interesting asymmetry between the $B$ and $I$ distributions after redshift 5 must be taken with caution owing to the small number of elements in those bins. Symbols are slightly offset but refer to the same redshift bin.

$z>5$, the bins are underpopulated. Thus the distribution asymmetry might be due to specific objects, as well as to the lack of consideration to the Lyman- $\alpha$ attenuation.

The inclusion of the Lyman- $\alpha$ forest effects and a more complete form of the fundamental transformation stated in Eq. (1) have recently been pointed out and reviewed by Andrei et al. (2014) and Coelho et al. (2014), which propose a new approach for calculating the absolute magnitudes (Coelho et al., in prep.). This new approach is planned for the next issues of the LQAC.

\section{Determination of morphological indexes}

The aspects of morphology and astrometric variability were crucial for selecting the quasars to form the successive versions of the ICRF, which were based on radio VLBI observations, as for the last one, the ICRF2 (Ma et al. 2009). Also in the optical band, these two aspects are increasingly addressed in programs of high astrometric precision. For the Gaia mission, the morphological indexes used here were included in the Gaia Initial QSO Catalog (GIQC, Andrei et al. 2012).

In radio waves bright spots are commonly formed where shocks traveling up the jets exchange energy with the external medium (Begelman et al. 1984) and are often taken as the radio centroid. In contrast to this, light emitted in (and around) the optical window originates mostly in the very central quasar, either by thermic processes in the accretion disk or relativistic processes at the basis of the jets. But, as the original quasistellar like-object term reveals, light can also originate in the host galaxy and, though comparatively less, in emission lines and as a consequence of the heating of more distant surrounding dust.

The study of what interaction exists between quasars and their host galaxies is central to establishing the difference to more quiescent galaxies and to investigating the host stellar population and formation rate (Cai et al. 2014). The morphology of the host galaxy is thus important for understanding the spectral energy distribution (SED), out of the discrete radio flux, spectroscopic, and photometric measurements presented in the catalog. However a large difficulty in studying it comes from the hundred or a thousand times stronger light emission from the AGN itself.

A lot of effort has therefore been put into disentangling the central source and the galaxy isophotes by suitable modeling. This was indeed proposed in the formulation of the GIQC and is implemented for all quasars observations in the Gaia mission (Ducourant et al. 2014). At the same time, a single point spread function (PSF) fitting can be analyzed to characterize the extent of the influence of the host galaxy on the compound image, which is crucial for astrometric purposes, as well as to decide how much is to be gained or lost from a complicated modeling of the centroid.

The approach of analyzing the PSF of the quasar image in comparison with the local mean PSF of stars of similar magnitude is the same adopted in the former versions of the LQAC. The elements of comparison are the parameters SHARP, SROUND, and GROUND, determined by IRAF's DAOFIND on the Johnston $B, R$, and $I$ digitized plates of the DSS. The morphological indexes of each quasar are given by

$M_{\mathrm{PC}}=\left|P_{\mathrm{Q}}-\overline{P_{\mathrm{s}}}\right| / \sigma s$

where $M_{\mathrm{PC}}$ is the morphological index of quasar $\mathrm{Q}$ for the PSF parameter $\mathrm{P}$ in color $\mathrm{C}$, given in comparison to the mean value from the stars $\mathrm{s}$, and normalized by the stellar standard deviation $\sigma$.

There are 146398 new sources with morphological indexes determined. Adding to the ones from the previous LQAC-2 version, there are in total 291903 quasars for which the morphological indexes were calculated. As in the previous version, concerning the new determinations, we can observe that although we have a large majority of stellar-like objects, there is a significant proportion of quasars for which the PSF is disturbed by the otherwise not easily detectable host. Moreover, still as in the LQAC-2, we can note in Figs. 6 and 7 that the more the host becomes conspicuous, the more the observational wavelength shifts from $B$ to $I$.

For each of the newly determined 146398 objects, at least one good image exists, that is to say that at least one morphological index could be obtained. At last the whole set of nine morphological indexes could be determined for $59 \%$ of the entries.

The direct comparison with the same morphological indexes for the stars provides a sanity check to verify zero averages and standard deviations at about 0.9 for SHARP, 0.7 for SROUND, and 0.8 for GROUND, which are slightly smaller at $I$ than $B$, 


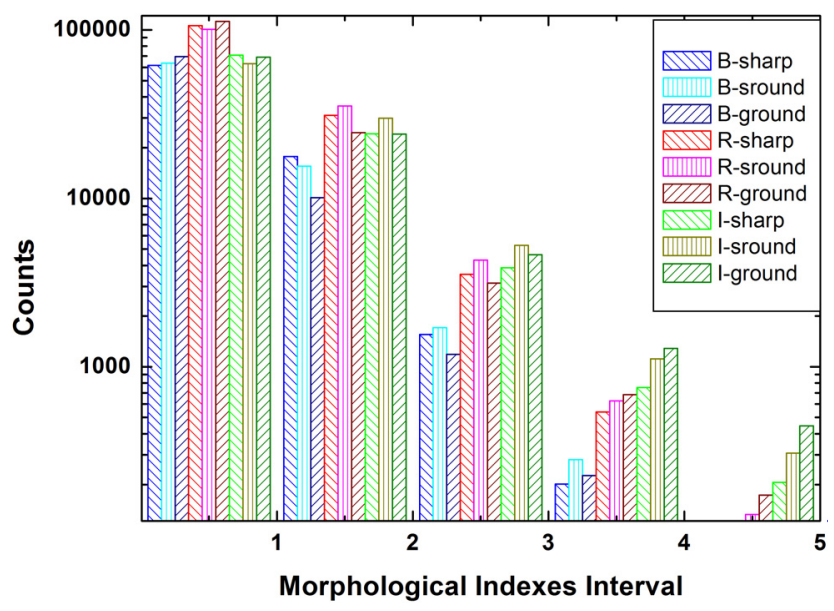

Fig. 6. Histogram of the morphological indexes for the quasars already belonging in the LQAC-2. The source count is on a $\log 10$ basis. Letters $B, R$, and $I$ refer to the three colors of DSS plates: blue, red, and infrared. The mnemonics after the letters refer to the IRAF's DAOFIND sharp, sround, and ground.

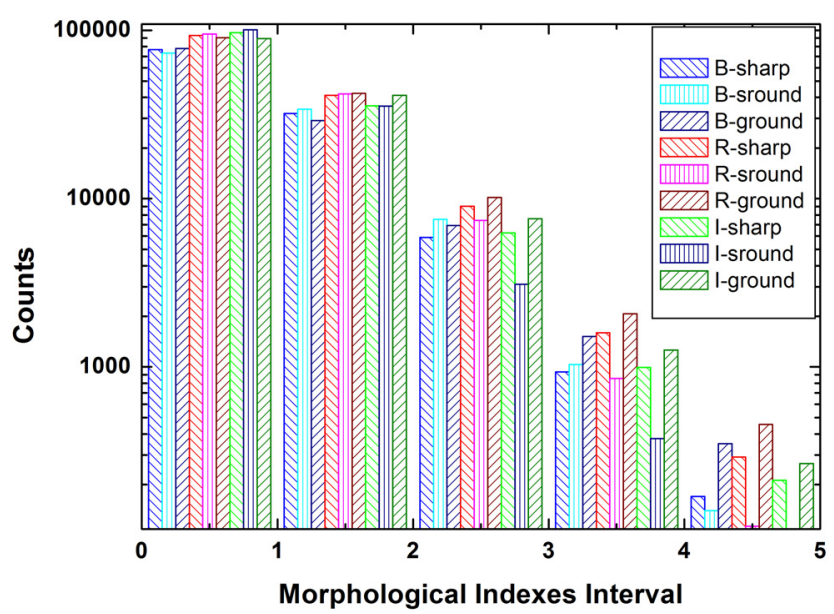

Fig. 7. Histogram of the morphological indexes for the new quasars in the LQAC3. The source count is on a $\log 10$ basis. Letters $B, R$, and $I$ refer to the three colors of the DSS plates: blue, red, and infrared. The mnemonics after the letters refer to the IRAF's DAOFIND sharp, sround, and ground.

but without statistically significant magnitude evolution between magnitudes $R=[9,22]$. DAOFIND has been largely and successfully used, for instance, for detecting clusters (Bastian et al. 2011). The way to circumvent its performance degradation in low illumination regimes is to use a logical training process (Mahonen \& Hakala 1995). This is done here by using the nearby, similar magnitude stars. Figure 8 shows the averages of the morphological indexes for the $B, R$, and $I$ plates, showing that although the underlying physical processes are quite complex, the general trend is coherent.

The main features of the morphological indexes for the new sources in the LQAC-3 are the same as in the former versions. However, the populations from which the sources are drawn present differences. We have already mentioned that the quasars newly included in the LQAC-3 come from the Baryon Oscillation Spectroscopic Survey (SDSS-III: BOSS, Ross et al. 2013). In contrast to the two former issues of the SDSS, where magnitudes peaked around 18 and the redshift range peaked around 1, the SDSS.III/BOSS was designed to target quasars in the redshift range $z=[2.2,3.5]$. This is a very relevant stretch of

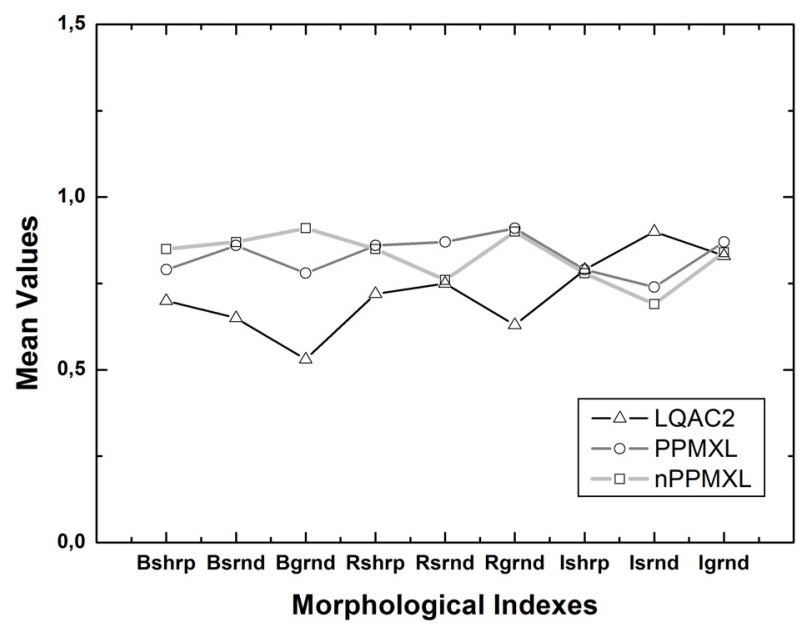

Fig. 8. Mean values of the morphological indexes. (Because of the large number of elements, the standard error's bar is invisible in this scale.) The line with triangles refers to the quasars already present in the LQAC-2. The line with circles refers to the new quasars in the LQAC3 that also are found in the PPMXL catalog. Correspondingly, the line with squares refers to the new LQAC3 quasars not found in the PPMXL. The new LQAC3 population is trimmed brighter than $R=19$.

the quasar luminosity function, when the quasar number density and luminosity were at their maximum. At the same time, the quasar luminosity function is such that increasing redshift is counterbalanced by increasing luminosity, leading to the dimming of apparent magnitudes being very attenuated. As the energy is fed back from the active nuclei to the host galaxy, the latter often presents as a massive elliptical type with large quantities of dust and a high rate of star formation, compatible with both infrared and UV emission. But its direct imaging is unlikely to be detected because of cosmological dimming of the surface brightness. In the BOSS description, the incompleteness due to the absence of quasars for which the galaxy emerges is said to be smaller than $3 \%$ in a regime brighter than magnitude $r=21$ (Ross et al. 2013). The SDSS photometric classifier that uses the amount of light within growing rings around the brighter central spot is well suited to selecting compact targets for spectroscopic measurements.

To maximize the certainty of the morphological indexes for the new, more distant sources included in the LQAC-3, we took three more conservative steps. We calculated the source count and convolve them with the Mahonen \& Hakala (1995) distribution what downsizes the indexes. We restricted the analysis to only sources brighter than $R=19$. We searched matches in the PPMXL catalog, limiting the candidates to those that are close in distance and in magnitude and that have a negligible proper motion. There are $65 \%$ more quasars found in the PPMXL than not (slightly less for the $B$ plates), and the PPMXL sources are on average $0.85 R$ magnitude brighter. By comparing the PPMXL and non-PPMXL found quasars, however, the correlation over all plates and morphological indexes is $99.9 \%$, thus clearly implying that the images on the DSS plates were found correctly and that the training of the morphological indexes made the magnitude effects unimportant. Finally we proceeded to examine the distribution indexes with respect to the redshift, shown in Fig. 9. As expected, stellar-like close-to-zero values are found, increasing from $B$ to $I$. Much less obviously, within each color the averages of the morphological indexes remain nearly constant, which would favor a scenario of coevolution of galaxies and supermassive black holes (Heckman \& Best 2014). 


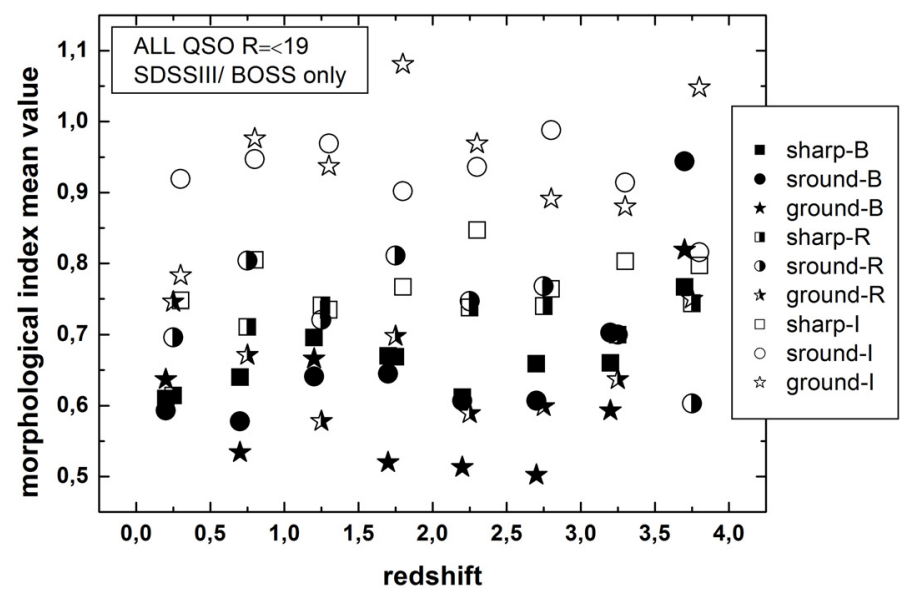

Fig. 9. Distribution of the morphological indexes with respect to the redshift. To avoid any contamination due brightness and distance, only sources with $R<19$ enter into the averages. Moreover, to avoid contamination from different selection criteria, the sources were selected just from the SDSS list. The color code is dark symbols for $B$, half colored symbols for $R$, and clear symbols for $I$. Symbols are slightly offset but refer to the same redshift bin. $B$ morphological indexes are offset to the left, and $I$ morphological indexes are offset to the right.

\section{Double quasars, coincident quasars, and false pairs}

Quasar pairs do not systematically characterize objects that are physically linked together. In fact we can distinguish three kinds of pairs of quasars for which it looks necessary to adopt a welldefined terminology. Thus we call "binary quasars" objects that are true physical associations. By contrast, we call "coincident" quasars two objects that have no physical link but whose lines of sights are very close. In addition we must consider "false pairs" consisting of the same quasar as has been recorded by two different catalogs with close coordinates. This last case supposes that for at least one of the two catalogs, the coordinates' offsets are larger than the radius used for our cross-identifications, typically $2^{\prime \prime}$, which means that its astrometric quality is highly questionable. A fourth category of quasar pairs can also be mentioned: the gravitationally lensed quasars, where the first example was discovered by Walsh et al. (1979) and whose a sample of 88 objects has been listed by Véron-Cetty \& Véron (2010).

A related study carried out during the construction of the first release of the LQAC (Souchay et al. 2009) has shown that the risk of finding false pairs is obviously zero for objects involving VLBI radio surveys, such as the ICRF (flag A), the $\operatorname{VLBA}(\mathrm{B})$, the $\operatorname{VLA}(\mathrm{C})$, and the JVAS(D) with milliarcsecond or sub-milliarcsecond accuracy. This risk is still basically zero for the SDSS(E), the $2 \mathrm{dF}(\mathrm{F})$, the $2 \mathrm{dF}-\mathrm{SDSS} \operatorname{LRG}(\mathrm{G})$ with subarcsecond accuracy. In contrast, the risk of a false pair is not negligible when the identification for one of the two quasars concerned has been done with the FIRST $(\mathrm{H})$ or the Hewitt and Burbridge(I) surveys, as well as with some small catalogs compiled by Véron-Cetty \& Véron (2010), which have shown some coordinate uncertainty at the level of 1 arcsec.

Gathering quasars from different surveys in the same cata$\log$ as the LQAC-3 constitutes a good opportunity to search for pairs of quasars. Their nature and distribution can have some cosmological implications, as shown by Hennawi et al. (2006), who present a detailed study of quasar pairs conducted from the SDSS and 2dF QSO redshift survey. They find a sample of 221 new pairs with proper transverse separations smaller than

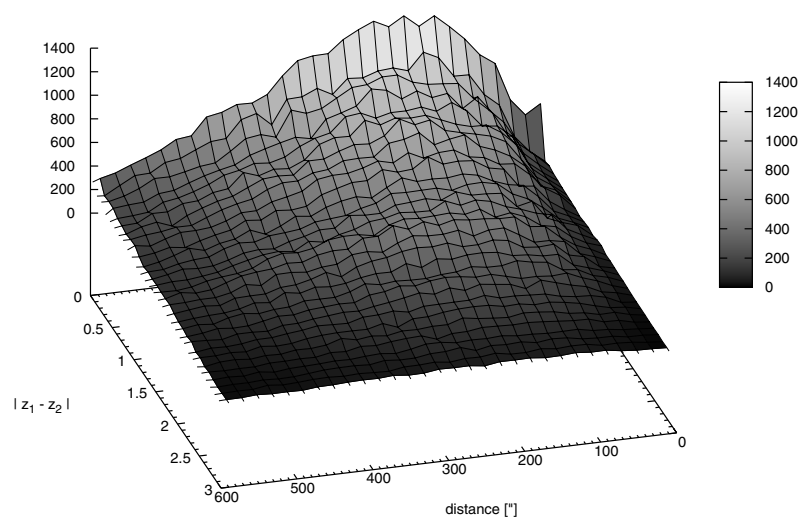

Fig. 10. 2D histogram showing the total number of quasars pairs in the LQAC-3 catalog with respect to their angular distance $\rho$ and their redshift difference $\Delta z$. Bin intervals are 20" for $\rho$ and 0.1 for $\Delta z$.

a threshold of $1 h^{-1} \mathrm{Mpc}$ over the redhift range of $0.5<z<$ 3.0. In particular, they noticed a small-scale excess of double quasars, which can probably be attributed to dissipative intercation events.

A specific study of quasar pairs, in particular intended to classify them in one of the three categories above (binary quasars, coincident quasars, false pairs), was done by Gattano et al. (2014) starting from the 188504 objects of the LQAC-2 release, both by using real images of the objects, when available, and by taking their redshifts into account. For instance, a significant difference in redshift value clearly supports the presence of a pair of coincident quasars, which in some cases is firmly confirmed by two neighboring objects in the field at the predicted position. In contrast, close values of redshifts with a small angular separation favor the two other cases, i.e. a false pair or a binary quasar. A visual check may clearly determine which case should be retained.

Such a study is scheduled in the scope of an ongoing paper devoted to detecting quasar pairs and to confirming their status as double quasars, coincident quasars, or false pairs, starting from the large LQAC-3 sample. Nevertheless, as a first approach we show in Fig. 10 the 2D histogramm of the number of quasars pairs found in each bidimensional bin $(\Delta z, \rho)$ where $\Delta z$ is the redshift difference and $\rho$ is the angular distance between the two components. Figure 11 represents the same histogram but by keeping only the pairs of quasars both belonging to the dominant SDSS survey, whereas in Fig. 12 we show only the pairs for which at least one of the components does not belong to the SDSS.

The three histograms show that for any angular distance $\rho$, the largest number of quasar pairs is obtained for the first bin $0<\Delta z<0.1$. For the overall sample (Fig. 10), this number peaks at 1381 pairs for $180^{\prime \prime}<\rho<200^{\prime \prime}$. For the exclusive SDSS pairs (Fig. 11), the peak is 1149 for the same interval in $\rho$, whereas for the pairs involving at least a non SDSS object (Fig. 12), we can observe two peaks: one at 335 pairs for the lowest bin value $0^{\prime \prime}<\rho<20^{\prime \prime}$, and another one at 320 pairs for $120^{\prime \prime}<\rho<140^{\prime \prime}$. The definite presence of the first peak is doubtful: it should be explained by a dominant number of false pairs resulting from the fact that at least one of the components comes from a catalog with poor astrometric performance. This will be investigated in the forthcoming paper mentioned above. 


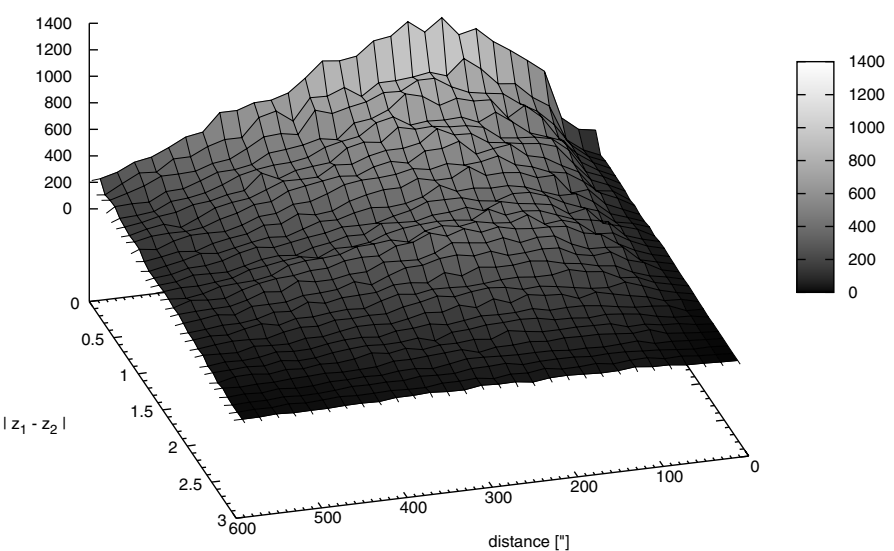

Fig. 11. 2D histogram showing the total number of SDSS quasars pairs in the LQAC-3 catalog with respect to their angular distance $\rho$ and their redshift difference $\Delta z$. Bin intervals are 20" for $\rho$ and 0.1 for $\Delta z$.

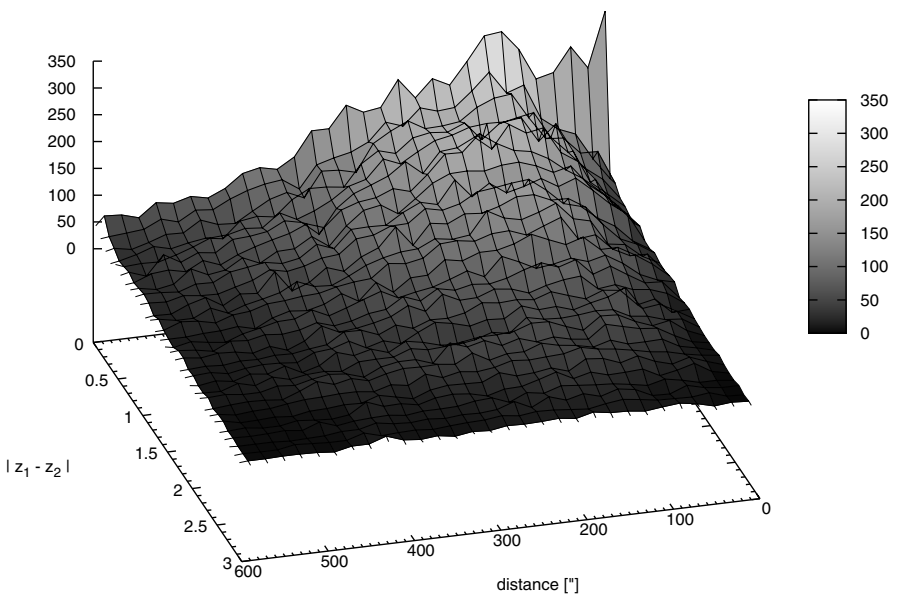

Fig. 12. 2D histogram showing the number quasars pairs with at least one non SDSS component in the LQAC-3 catalog with respect to their angular distance $\rho$ and their redshift difference $\Delta z$. Bin intervals are $20^{\prime \prime}$ for $\rho$ and 0.1 for $\Delta z$.

\section{Presentation of the LQAC-3}

The LQAC-3 gathers a total of 321957 objects. For a very large part, they include quasars but with a small proportion of active galaxies and BL Lac objects, already present in the last Véron-Cetty \& Véron (2010) compilation.

\subsection{Data and format}

In the following we present the items of the LQAC-3 in a sequential order according to each column.

- Column 1 gives the LQAC number of the object. This number was constructed starting from boxes defined from intervals in degrees both in right ascension and in declination. For instance, the quasar named LQAC_313-034_002 is the second one included in the box corresponding to intervals $313^{\circ}<\alpha<314^{\circ}$ and $-35^{\circ}<\delta<-34^{\circ}$. For objects already present in the LQAC-2 release, the number remains unchanged. Moreover, some SDSS quasars present in the LQAC-2 compilation were no longer present in the LQAC-3 one. In that case, for the sake of coherence, their number has not been utilized again to name a new quasar in the same box.
- Column 2 gives the original name of the quasar. For objects already present in the catalog of quasars and active nuclei by Véron-Cetty \& Véron (2010), we use the same terminology. This column was not present in the LQAC and LQAC-2 catalogs.

- Column 3 gives a flag that indicates the attribution of the nature of the object: "QSO" for quasars fully reckoned optically, "AGN" for AGN's, "BLA" for "BLAC", and "RAD" for point-like objects concerned principally with radio VLBI emissions (catalogs A, B, C, D), which are those for which we have no optical counterpart in our compilation.

- Columns 4 and 5 are the equatorial coordinates $(\alpha, \delta)$ of the object given by the original catalog.

- Columns 6 and 7 are the equatorial coordinates $(\alpha, \delta)$ of the object in the ICRF according to the astrometric algorithms described in Sect. 4. These coordinates are supposed to be more exact than the original ones except for the quasars present in the radio VLBI catalogs (flags A, B, C, D).

- Columns 8 to 20 provide letter codes to indicate the presence of the quasar in one of the 13 catalogs from $\mathrm{A}$ to $\mathrm{M}$ as given by the nomenclature in Table 1, the flag "E" representing the SDSS. All information related to these catalogs is given by Souchay et al. (2012).

- Column 21 gives the redshift as determined from the original catalog generally indicated in Col. 2 .

- Columns 22 to 28 are the visual magnitudes listed by increasing value of the wavelength. The photometric bands are the $u, b, v, g, r, i, z$, respectively. The photometric system in which the magnitude has been measured is not homogeneous for each band. The priority is indicated by the first flag starting from the first optical catalog, the SDSS, with flag E. For instance, the value of the $r$ magnitude of a quasar observed both by the SDSS (flag E) and the 2QZ (flag F) is taken from the SDSS, whereas the value of the $b$ magnitude, only present in the 2QZ, will be taken from this survey.

- Columns 29 and 30 present the $J$ and $K$ infrared magnitudes for 21844 quasars for which we have found a counterpart in the 2MASS survey.

- Columns 31 to 35 give the radio fluxes at $1.4 \mathrm{GHz}(20 \mathrm{~cm})$, $2.3 \mathrm{GHz}(13 \mathrm{~cm}), 5.0 \mathrm{GHz}(6 \mathrm{~cm}), 8.4 \mathrm{GHz}(3.6 \mathrm{~cm})$, and $24 \mathrm{GHz}(1.2 \mathrm{~cm})$, respectively, when available. These radio fluxes concern exclusively the quasars coming from the four first catalogs (flags A to D), those coming from VLBI sessions.

- Columns 36 to 44 provide us with the morphological indexes where the determination has been explained in full detail in Sect. 5.4. They concern the $B$ (Cols. 35 to 37 ), $R$ (Cols. 38 to 40 ), and $I$ (Cols. 41 to 43 ) bands with three indexes each, the first one characterizing the skewness, the second one the roundness, and the last the normality.

\section{References}

Aihara, H., Prieto, C. A., An, D., et al. 2011, ApJS, 193, 1

Andrei, A. H., Souchay, J., Zacharias, N., et al. 2009, A\&A, 505, 385

Andrei, A. H., Anton, S., Barache, C., et al. 2012, in SF2A-2012: Proc. Annual meeting of the French Society of Astronomy and Astrophysics, 61

Andrei, A. H., Coelho, B., \& Antón, S. 2014, in Journées Systèmes de Référence Spatio-Temporels, St. Petersburg meeting

Bastian, N., Adamo, A., Gieles, M., et al. 2011, MNRAS, 417, L6

Begelman, M. C., Blandford, R. D., \& Rees, M. J. 1984, Rev. Mod. Phys., 56, 255

Bolton, A. S., Schlegel, D. J., Aubourg, E., et al. 2012, AJ, 144, 144

Cai, Z., Fan, X., Noterdaeme, P., et al. 2014, ApJ, 739, 139

Coelho, B., Andrei, A. H., \& Antón, S. 2014, EGSG Workshop, Meudon, Dec. $8-10$ 
J. Souchay et al.: The third release of the Large Quasar Astrometric Catalog (LQAC-3)

Ducourant, C., Krone-Martins, A., Galluccio, L., \& Teixeira, R. 2014, in SF2A-2014: Proc. Annual meeting of the French Society of Astronomy and Astrophysics, 421

Gattano, C., Souchay, J., \& Barache, C. 2014, A\&A, 564, A117

Komatsu, E., Smith, K. M., Dunkley, J., et al. 2011, ApJS, 912, 18

Heckman, T. M., \& Best, P. N. 2014, ARA\&A, 52, 589

Hennawi, J. F., Strauss, M. A., Oguri, M., et al. 2006, AJ, 131, 1

Hogg, D. W. 2000, ArXiv e-prints [arXiv: astro-ph/9905116]

Lasker, B. M., Lattanzi, M. G., Mc. Lean, B. J., et al. 2008, AJ, 136, 735

Ma, C., Arias, E. F., Bianco, G., et al. 2009, IERS Technical Note, 35, 1

Mahonen, P. H., \& Hakala, P. J. 1995, ApJ, 452, L77

Morgan, C. W., Kochanek, C. S., Morgan, N. D., \& Falco, E. E. 2010, ApJ, 712, 1129

Monet, D. G., Levine, S. E. C. B., Ables, H. D., et al. 2003, AJ, 125, 984

Pâris, I., Petitjean, P., Aubourg, E., et al. 2012, A\&A, 548, A66
Pâris, I., Petitjean, P., Aubourg, E., et al. 2014, A\&A, 563, A54

Richards, G. T., Lacy, M., Storrie-Lombardi, L. J., et al. 2006, AJ, 131, 2766

Ross, N. P., McGreer, I. D., White, M., et al. 2013, ApJ, 773, 14

Schlegel, D. J., Finkbeiner, D. P., \& Davis, M. 1998, ApJ, 500, 525

Schneider, D. P, Richards, G. T., Hall, P. B., et al. 2010, AJ, 139, 2360

Shen, Y., Greene, J. E., Strauss, M. A., Richards, G. T., \& Schneider, D. P. 2008, ApJ, 680, 169

Souchay, J., Andrei, A. H., Barache, C., et al. 2009, A\&A, 494, 815

Souchay, J., Andrei, A. H., Barache, C., et al. 2012, A\&A, 537, A99

Véron-Cetty, M. P., \& Véron, P. 1984, A\&A, ESO Scientific Report, No. 1

Véron-Cetty, M. P., \& Véron, P. 2006, A\&A, 455, 773

Véron-Cetty, M. P., \& Véron, P. 2010, A\&A, 518, A10

Walsh, N. E., Carswell, R. F., \& Weymann, R. J. 1979, Nature, 279, 381

Zacharias, N., Urban, S. E., Zacharias, M. I., et al. 2004, AJ, 127, 3043 